\title{
Immunoglobulin G4-Related Ophthalmic Disease Involving the Sclera Misdiagnosed as Intraocular Tumor: Report of One Case
}

\author{
Hiroshi Goto Shunichiro Ueda \\ Department of Ophthalmology, Tokyo Medical University, Tokyo, Japan
}

\author{
Key Words \\ Immunoglobulin G4-related ophthalmic disease . \\ Intraocular tumor - Sclera - Transscleral biopsy . \\ Histopathology
}

\begin{abstract}
Aims: Immunoglobulin G4 (lgG4)-related disease is known to have diverse clinical manifestations. We report a case of lgG4-related ophthalmic disease misdiagnosed as intraocular tumor. Methods: Case report. Results: A 41-year-old man was referred to our department with a diagnosis of intraocular tumor, presumably choroidal melanoma. Although MR imaging was compatible with choroidal melanoma, he had been observed periodically without treatment because fundus findings were not typical of melanoma and visual function was preserved. He was again referred to us 15 years later due to increased tumor size and visual field disturbance. Transscleral biopsy of the intraocular tumor was attempted to make a histopathological diagnosis. During the biopsy procedure, thick placoid tissue adhering to the sclera was found at a location consistent with the site of the elevated intraocular lesion. Histopathological examination of this extraocular material revealed proliferation of dense fibrous tissue with follicular proliferation of lymphocytes and plasmacytes. The plasmacytes were diffusely positive for $\lg G$ and IgG4. Serum IgG4 level was slightly elevated and no extra-
\end{abstract}

ocular lesion was detected. Despite administration of systemic corticosteroids after biopsy, the intraocular mass was not reduced. Visual acuity of $20 / 20$ has been maintained as of the last follow-up. Conclusion: IgG4-related disease should be included in the differential diagnosis of intraocular elevated lesion.

(c) 2016 S. Karger AG, Basel

\section{Introduction}

Immunoglobulin G4 (IgG4)-related disease is a clinical entity characterized by infiltration of IgG4-immunopositive plasmacytes and elevated serum IgG4 concentration accompanied by mass formation in various organs including the lacrimal gland, salivary gland, and pancreas. Recent studies have confirmed that conditions previously diagnosed as Mikulicz disease as well as various types of lymphoplasmacytic infiltrative disorders are consistent with a diagnosis of IgG4-related disease $[1,2]$. After the establishment of comprehensive diagnostic criteria for IgG4-related disease in 2012 [3], the diagnostic criteria for IgG4-related ophthalmic disease (IgG4-ROD) have recently been reported, based on both clinical and histopathologic features of ocular lesions [4]. We report a unique case of IgG4-ROD involving the sclera, which had been misdiagnosed as intraocular tumor for over 10 years.

\section{KARGER}

E-Mail karger@karger.com

www.karger.com/oop
(C) 2016 S. Karger AG, Basel

2296-4681/16/0024-0285\$39.50/0
Hiroshi Goto, MD

Department of Ophthalmology, Tokyo Medical University

6-7-1 Nishi-shinjuku, Shinjuku-ku

Tokyo 160-0023 (Japan)

E-Mail goto-1115@ tokyo-med.ac.jp 

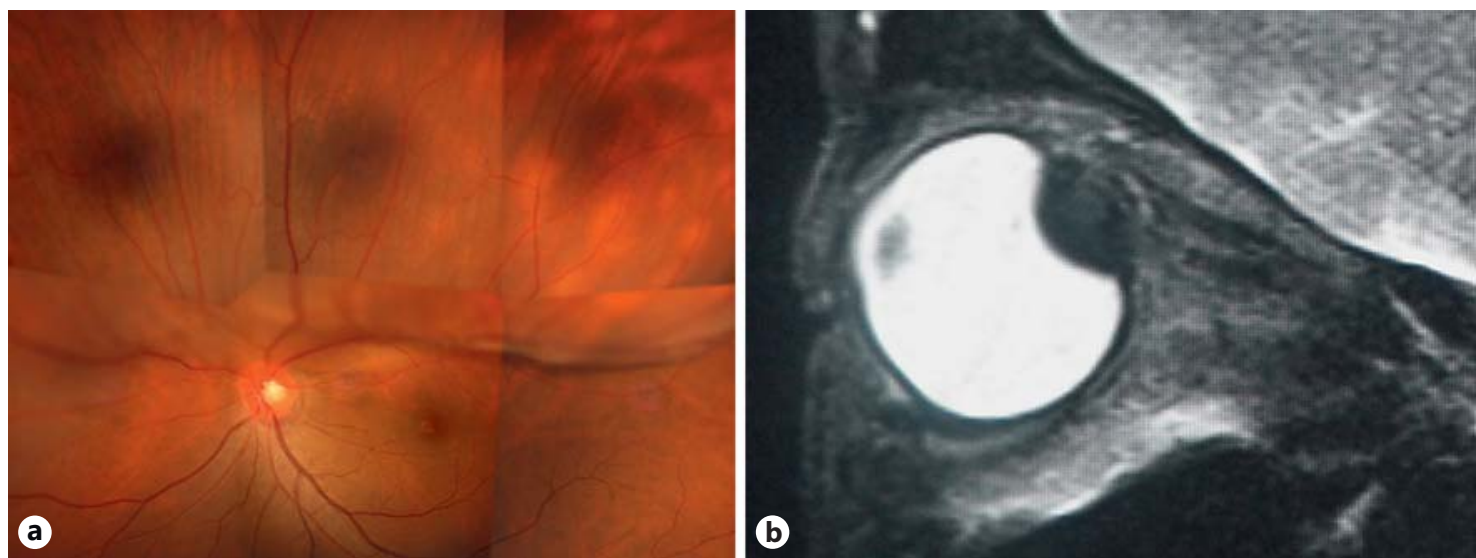

Fig. 1. a Ocular fundus photograph shows an elevated lesion in the superior region extending from the optic disc. b T2-weighted MR image depicts an intraocular mass with low intensity.
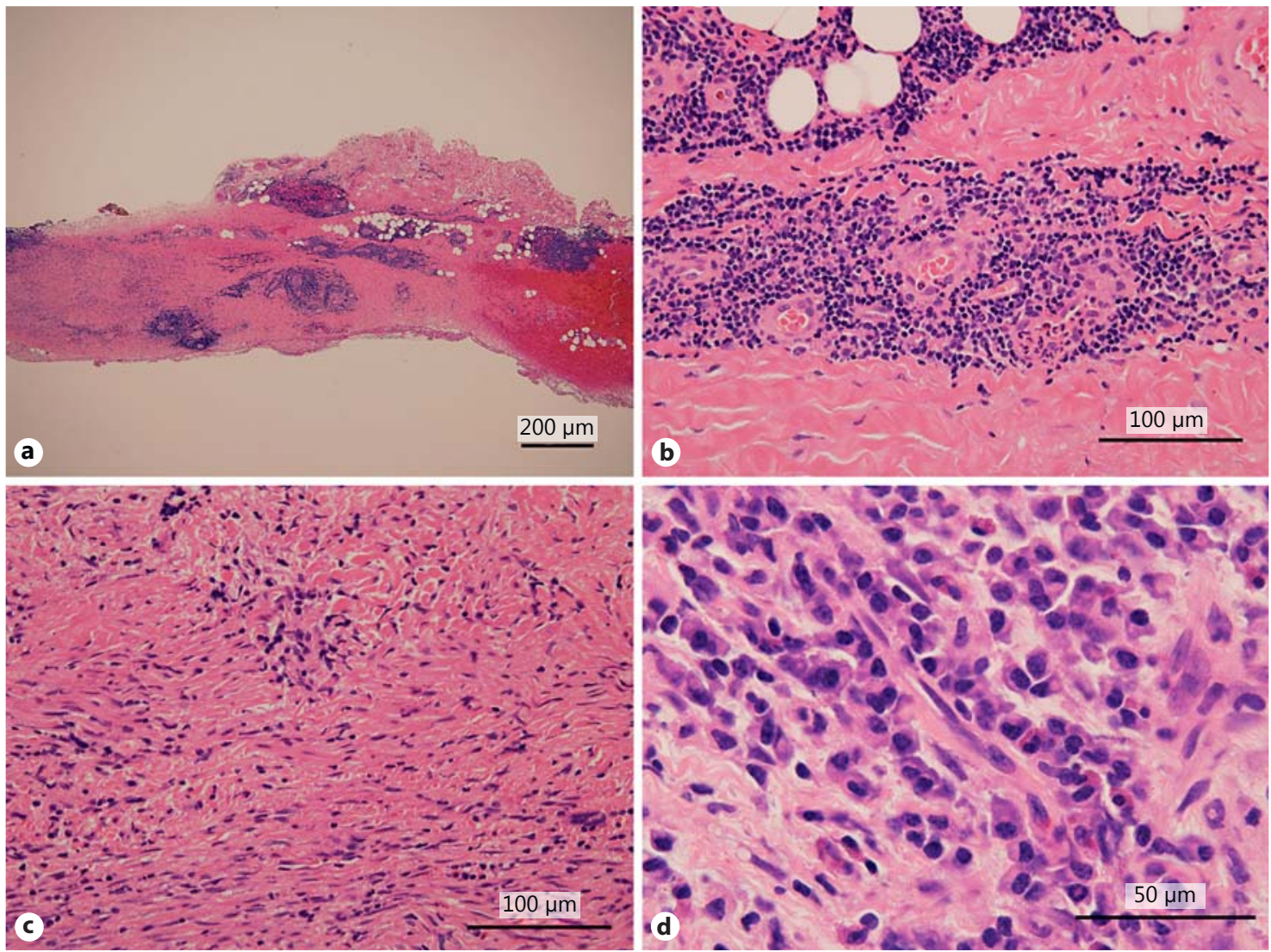

Fig. 2. Histopathology of the excised episcleral placoid tissue (hematoxylin-eosin). a Low-magnification micrograph shows proliferation of dense fibrous tissue containing follicular cell infiltration $(\times 4)$. $\mathbf{b}$ The majority of the excised material is fibrous tissue $(\times 20)$. c Foci of infiltrative cells are present among the fibrous tissue $(\times 20)$. d Infiltrative cells consist of lymphocytes and plasmacytes $(\times 40)$. 


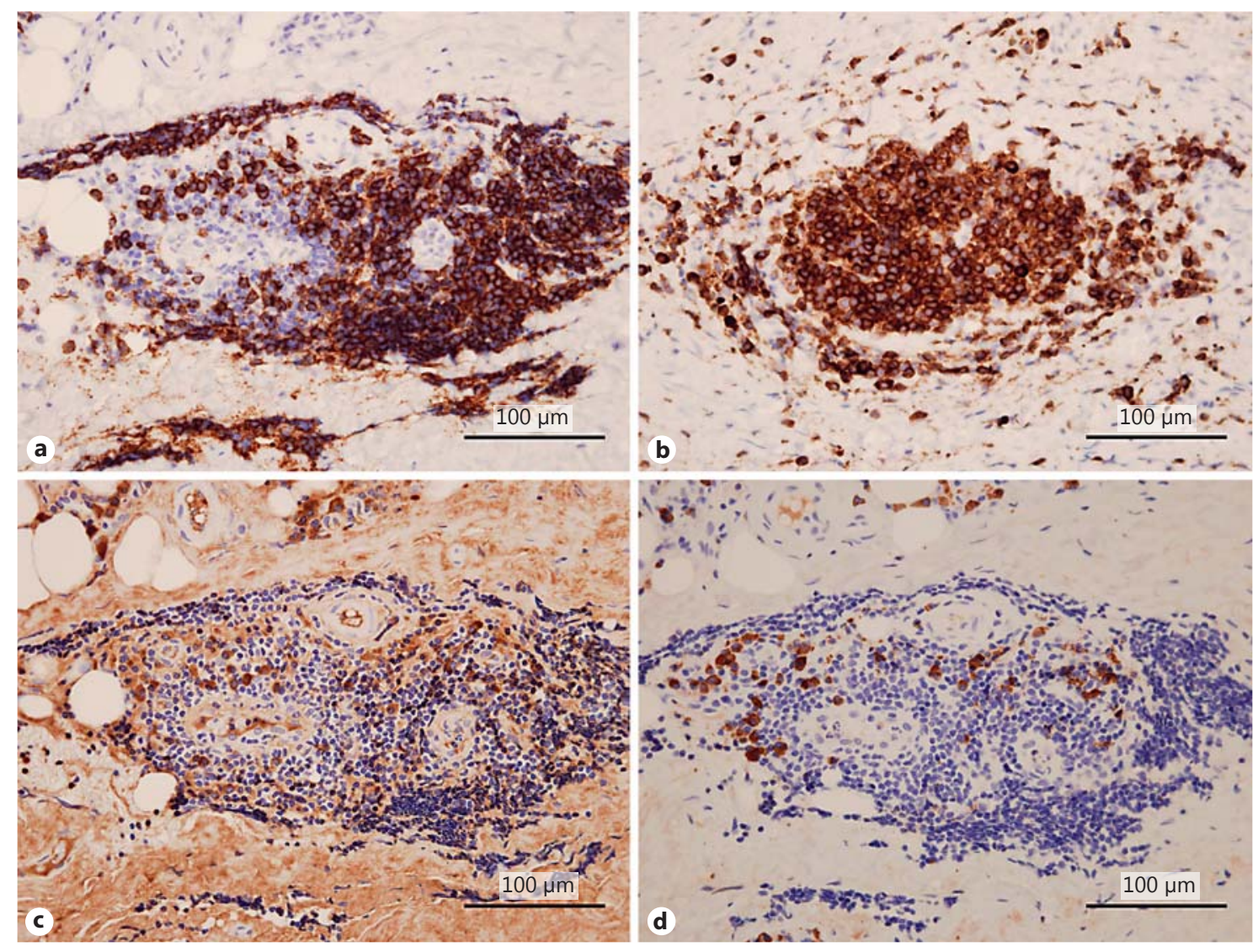

Fig. 3. Histopathology of the excised episcleral placoid tissue (immunohistochemical staining). a Lymphoplasmacytes infiltrating the fibrous tissue are diffusely positive for CD20 $(\times 20)$. $\mathbf{b}$ CD79a reactivity is mainly found in follicular areas $(\times 20)$. c Scattered IgG-positive cells are observed $(\times 20)$. d IgG4 staining shows over 50 IgG4positive cells in a high power field. Approximately $40 \%$ of IgG-positive cells are positive for IgG4 $(\times 20)$.

\section{Case Report}

A 41-year-old man was referred to the Department of Ophthalmology, Tokyo Medical University Hospital in December 1998 with a clinical diagnosis of intraocular tumor, presumably choroidal melanoma. His best corrected visual acuity (BCVA) was 20/20 OU. Slitlamp examination revealed no significant findings in the anterior segment of both eyes. Fundus examination of his left eye showed an intraocular elevated lesion with normal fundus color, from the superior to nasal side of the optic disc. MR imaging depicted high intensity on T1-weighted image and low intensity on T2-weighted image. The results of systemic workup including gallium-67 scintigraphy were normal. Although MR imaging was compatible with a diagnosis of choroidal melanoma, the patient was observed periodically without treatment because fundus findings were not typical of melanoma. Ocular fundus examination as well as ultrasonography showed no significant changes for several years.

He was referred to us again in June 2013, 15 years after the initial visit, because of subjective visual field disturbance. His BCVA was $20 / 20$ OS. The elevated lesion in his fundus had apparently enlarged (fig. 1). Single photon emission computed tomography using $\mathrm{N}$-isopropyl-p-[ $\left.{ }^{123} \mathrm{I}\right]$ iodoamphetamine, which has been used for diagnosis of uveal melanoma [5], was negative. Biopsy of the intraocular lesion from the sclera was scheduled to make a histopathological diagnosis. Under local anesthesia, the bulbar conjunctiva was dissected from the superior limbus. Exposure of the superior rectus muscle was difficult because the Tenon capsule adhered to the sclera. Thick and yellowish-white placoid tissue around the sclera was observed, at a location consistent with the site of the elevated intraocular lesion. Some pieces of the placoid tissue were excised and examined histopathologically.

Microscopic examination revealed dense fibrous tissue containing follicular proliferation of lymphocytes and plasmacytes (fig. 2). Immunohistochemical staining showed that the lymphoplasmacytic cells were diffusely positive for CD20 as well as CD79a. Plasmacytes were positive for IgG, and over $40 \%$ of these cells (absolute count: over 55 cells per high power field) were positive for IgG4. Obliterative phlebitis was not present (fig. 3). Based on these histopathological findings, this case was diagnosed as IgG4-ROD involving the sclera.

Systemic workup carried out after biopsy revealed serum IgG4 of $120 \mathrm{mg} / \mathrm{dl}(4.8-105 \mathrm{mg} / \mathrm{dl})$ and IgG of $1,821 \mathrm{mg} / \mathrm{dl}\left(870_{-}\right.$ 
$1,700 \mathrm{mg} / \mathrm{dl})$. There were no signs of systemic involvement including the lacrimal gland, salivary gland, and pancreas.

Systemic administration of prednisolone was started, but the height and area of the intraocular elevated lesion did not change. Although supraduction restriction in the left eye persisted, the patient did not perceive diplopia in any direction. Visual acuity of 20/20 was maintained for 2 years after biopsy.

\section{Discussion}

Patients with symmetric enlargement of the lacrimal and salivary glands, who were diagnosed as Mikulicz disease in the past, were found to have abnormally high serum IgG4 levels [1]. Similar cases had been reported subsequently by many facilities [4]. It is now known that IgG4-related ophthalmic lesions are not limited to the lacrimal glands but may manifest with various patterns in the orbit and ocular tissues such as extraocular muscles, orbital nerve, and eyelid [6].

On the contrary, only a few cases of IgG4-ROD with scleral involvement have been reported $[7,8]$. Ohno et al. [7] reported a case of a 49-year-old patient who underwent enucleation of the eyeball with a clinical diagnosis of choroidal tumor. This case was first treated as scleritis, but was refractory to treatment with systemic corticosteroids and cyclosporine. The present case did not show any symptoms of anterior scleritis, but some clinical manifestations were compatible with posterior scleritis.

Histopathological examination of the episcleral material depicted foci of lymphoplasmacytic infiltration with IgG4-positive cells in the dense fibrous tissue. A possible pathogenesis of this case is expansion of lymphoplasma- cytic proliferation in episcleral tissue toward the eyeball resulting in an intraocular elevated lesion with progressive fibrotic change over time. Serum IgG4 level measured after biopsy was slightly elevated, but lower than that in diagnostic criteria [3]. The clinical, laboratory and histopathological findings of the present case deviate somewhat from typical cases of IgG4-ROD, presumably due to the long time lag before the diagnostic evaluations were performed.

Systemic corticosteroids are the established treatment for IgG4-related disease [9]. Although the present case did not respond to systemic corticosteroids, it is possible that the same treatment at the initial stage may have been effective to control disease activity. Immunosuppressive agents such as cyclosporine and monoclonal antibodies such as rituximab were not considered in this case after discontinuation of corticosteroids, because histopathological findings of biopsy specimens showed mostly fibrous tissue. IgG4-ROD should be included in the differential diagnosis of intraocular elevated lesion to avoid unnecessary aggressive treatments such as enucleation and radiation therapy.

\section{Statement of Ethics}

The patient gave informed consent for using his clinical information and presenting this case at a meeting or publishing it in a journal. Therefore, institutional ethical approval is not necessary.

\section{Disclosure Statement}

The authors declare no conflicts of interest and no financial interests.

\section{References}

1 Yamamoto M, Ohara M, Suzuki C, Naishiro Y, Yamamoto H, Takahashi H, et al: Elevated IgG4 concentrations in serum of patients with Mikulicz's disease. Scand J Rheum 2004;33: 432-433.

2 Japanese study group of IgG4-related ophthalmic disease: A prevalence study of IgG4related ophthalmic disease in Japan. Jpn J Ophthalmol 2013;57:573-579.

3 Umehara H, Okazaki K, Masaki Y, Masaki Y, Kawano M, Yamamoto M, et al: Comprehensive diagnostic criteria for IgG4-related disease (IgG4RD), 2011. Mod Rheumatol 2012; 22:21-30.
4 Goto $\mathrm{H}$, Takahira M, Azumi A, et al: Diagnostic criteria for IgG4-related ophthalmic disease. Jpn J Ophthalmol 2015;59:1-7.

5 Goto H, Usui M, Ishii I: Efficacy of (123)Nisopropyl-p-[(123)I]-iodoamphetamine single photon emission computed tomography for the diagnosis of uveal malignant melanoma. Am J Ophthalmol 2001;132: 937-939.

6 Sogabe Y, Ohshima K, Azumi A, Takahira M, Kase S, Tsuji $\mathrm{H}$, et al: Location and frequency of lesions in patients with IgG4-related ophthalmic diseases. Graefes Arch Clin Exp Ophthalmol 2014;52:531-538.
7 Ohno K, Sato Y, Ohshima K, et al: IgG4-related disease involving the sclera. Mod Rheumatol 2014;24:195-198.

8 Philippakis E, Cassoux N, Charlotte F, et al: IgG4-related disease masquerading as recurrent scleritis and chronic conjunctivitis. Ocul Immunol Inflamm 2015;23:168-172.

9 Khosroshahi A, Wallace ZS, Crowel JL, et al: International consensus guidance statement on the management and treatment of IgG4related disease. Arthritis Rheumatol 2015;67: 1688-1699. 